\title{
Systematic Evaluation of the Thermo-magnetic Properties of Nanoparticles Coated with PNIPAM
}

Pedro-A. Hernandez-A. ${ }^{1,2}$, Nadia. Garcia-F. ${ }^{2}$, Jorge-L. Iriqui-R. ${ }^{1}$, Hiram Higuera-V. ${ }^{1}$, Eleazar León-S ${ }^{1}$, Reynaldo Esquivel $^{2}$, Iván E. Moreno-Cortez ${ }^{3}$ and M.E. Álvarez-Ramos ${ }^{2}$

${ }^{1}$ Universidad Estatal de Sonora, Hermosillo, Sonora, Mexico, ${ }^{2}$ Universidad de Sonora, Hermosillo, Sonora, Mexico, ${ }^{3}$ Universidad Autónoma de Nuevo León, Monterrey, Nuevo Leon, Mexico

Recent publications have reported important advances in the development of new smart nano-platforms due to their response when exposed to external physical and chemical changes, leading to a dramatic modification of their properties. These features allow the potential use of these smart materials in the field of therapeutic biomedicine [1, 2]. In this research, a systematic evaluation of the synthesis parameters of a multi-responsive core-shell nanoplatform $\left(\mathrm{Fe}_{3} \mathrm{O}_{4}\right.$ nanoparticles coated by a poly $(\mathrm{N}-$ isopropylacrylamide) matrix) was performed. These nanocomposites allow the possibility of controlling certain properties, such as particle size and morphology. The polymer coating was performed by in-situ free radical polymerization of $\mathrm{N}$-isopropylacrylamide on $\mathrm{Fe}_{3} \mathrm{O}_{4}$ nanoparticles to obtain a nanocomposite with core-shell structure.

In our case, the magnetite NPs were coated with oleic acid to improve their stability and biocompatibility. The $\zeta$-potential of $\mathrm{Fe}_{3} \mathrm{O}_{4}$ NPs after the functionalization process is around $-33 \mathrm{mV}$, this coincides with previous works [3]. This study reveals that the addition of PNIPAM coating to $\mathrm{Fe}_{3} \mathrm{O}_{4} \mathrm{NPs}_{\text {induced a }}$ dramatic change in $\zeta$-potential. The nanoplatform exhibit values near to the isoelectric point, no precipitation or aggregation was observed even eight weeks after preparation. This result could be due to the steric effects of PNIPAM polymer chains [4]. Figure 1 presents the hysteresis loops of the nanocomposite. This phenomenon allows us to conclude that it was possible to obtain nanocomposites of magnetite with PNIPAM matrix through co-precipitation in situ, thus obtaining multi-responsive materials (magnetic-temperature) for possible potential applications in the biomedical area. Size and morphology of the nanocomposite were evaluated by scanning electron microscopy. Figure 2 illustrates that the nanoplatform have a size of around $350 \mathrm{~nm}$ and a spherical morphology. A core-shell structure is observed confirming what the previous characterizations have already suggested. A clearer comprehension of the role played by synthesis parameters in this platform was achieved. The expected characteristics for a potential use as a drug internationalization vector were also obtained. 


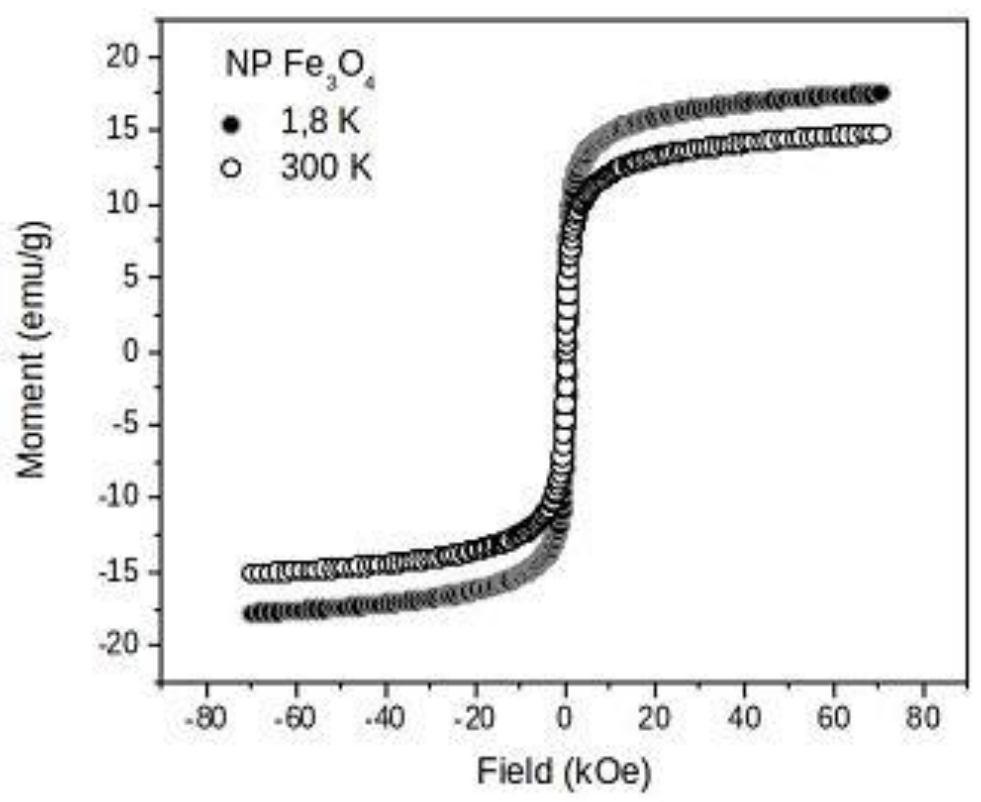

Figure 1. Magnetic curve of nanocomposite at 1.8 and $300 \mathrm{~K}$. The hysteresis loops were obtained with a maximumfield of $7 \times 104$ Oe. The samples were vacuum dried to be analyzed.

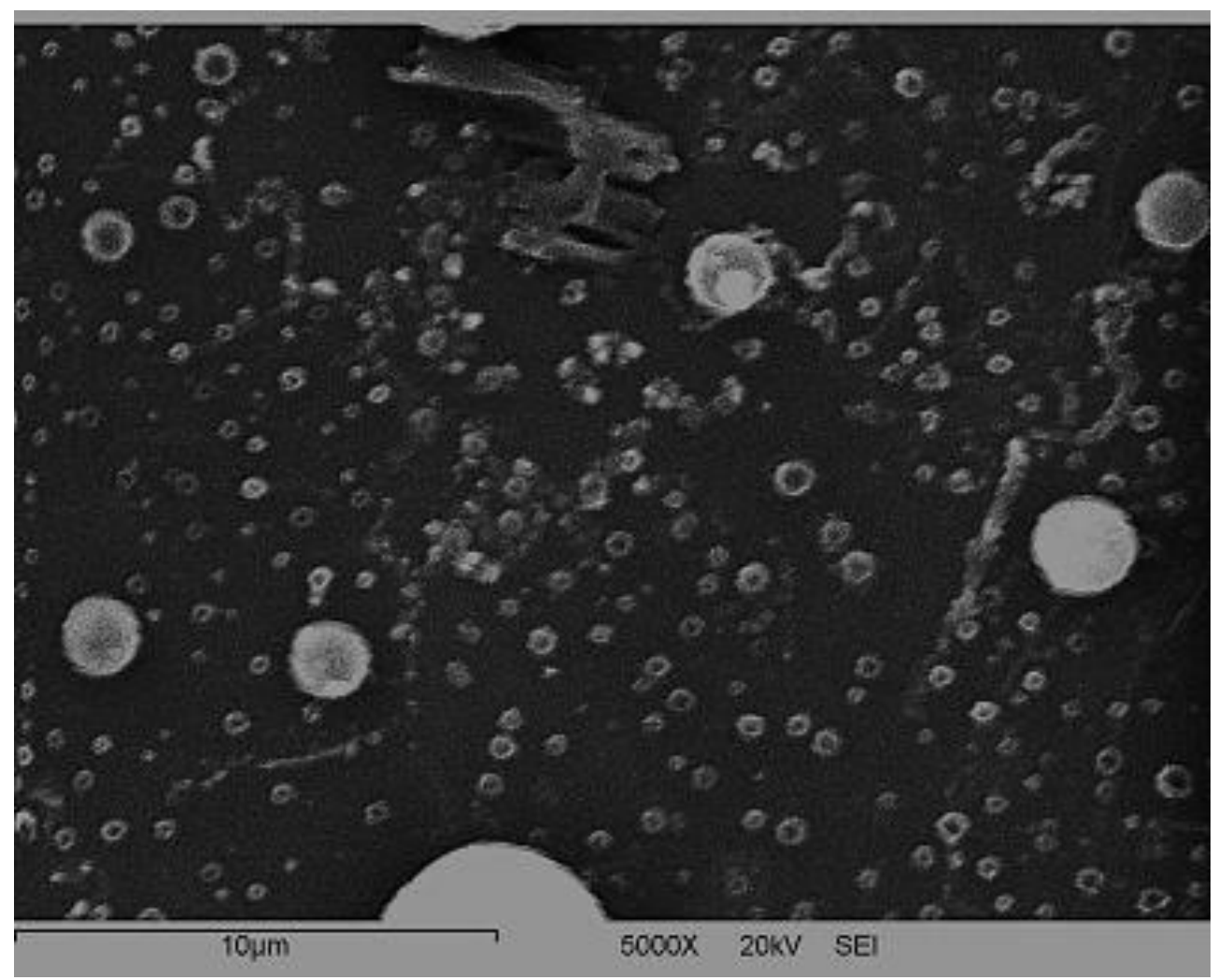

Figure 2. SEM micrography of nanocomposite, the nanoplatform exhibits core-shell characteristics, PNIPAM agglomerates are observed (scale bar $=10 \mu \mathrm{m})$. 


\section{References}

1. Wust, P. et. Al. Hyperthermia in combined treatment of cancer, The Lancet Oncology, Vol. 3, Issue 8, Pages 487-497, 2002.

2. Liu, J. et. Al. Poly(N-vinylcaprolactam): a thermoresponsive macromolecule with promising future in biomedical field. Adv. Healthc. Mater, vol 3, pages 1941-68, 2014.

3. Shete, P.B., Patil, R.M., Tiwale, B.M. and Pawar, S.H., 2018. Water dispersible oleic acid-coated Fe3O4 nanoparticles for biomedical applications. Journal of Magnetism and Magnetic Materials, 377, pp.406410.

4. Bradley, M. and Garcia-Risueño, B.S., 2011. Journal of colloid and interface science symmetric and asymmetric adsorption of $\mathrm{pH}$ - responsive gold nanoparticles onto microgel particles and dispersion characterisation. Journal of Colloid and Interface Science, 355(2), pp.321-327 